\title{
Frecuencia de parásitos gastrointestinales de perros en parques públicos de dos municipios vecinos del Estado de México
}

Frequency of gastronintestinal parasite of dogs in public parks in two neighboring municipalities of state of Mexico

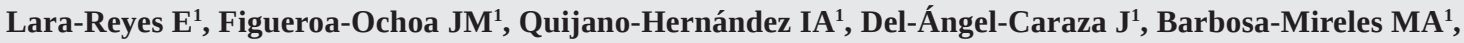
Victoria-Mora JM${ }^{1}$, Beltrán-León $T^{1}$

\section{Resumen}

La contaminación de suelos por parásitos gastrointestinales representa un problema mundial y un riesgo a la salud. Objetivo. Determinar la presencia de parásitos en heces de perros en los parques públicos del área. Materiales y métodos. Se recolectaron muestras de heces en 27 parques públicos de Metepec y Toluca Estado de México, mismos que se procesaron con 3 técnicas parasitológicas; $81.4 \%$ de los parques públicos resultaron positivos a parásitos gastrointestinales, con una frecuencia global de muestras del 16.5\%. El porcentaje de parasitosis con potencial zoonótico fue de $81.3 \%$; Toxocara spp, Ancylostoma spp y Giardia spp fueron las especies zoonóticas identificadas. Conclusiones. Los resultados indican que los parques de la zona conurbada de Toluca representan un problema de salud pública importante al ser una fuente de parásitos gastrointestinales zoonóticos de perro.

Palabras claves: Parasitosis, zoonosis, contaminación de áreas verdes

\section{Abstract}

Soil contamination by gastrointestinal parasites represents a global problem and a health risk. Objective. Determine the presence of parasites in dog feces in public parks in the area. Materials and methods. Stool samples were collected in 27 public parks in Metepec and Toluca Estado de México, which were processed with 3 parasitological techniques; $81.4 \%$ of public parks were positive for gastrointestinal parasites, with an overall sample rate of $16.5 \%$. The percentage of parasitosis with zoonotic potential was $81.3 \%$; Toxocara

1. Hospital Veterinario Pequeñas Especies de la Facultad de Medicina Veterinaria y Zootecnia de la Universidad Autónoma del Estado de México, Toluca, Estado de México, México.

Correspondencia: iaquijanoh@uaemex.mx

ORCID:

https://orcid.org/0000-0001-9273-6397 - Elizabeth Lara Reyes

https://orcid.org/0000-0001-9444-8225 - José Miguel Figueroa 0choa

https://orcid.org/0000-0003-2408-3827 - Israel Alejandro Quijano Hernández

https://orcid.org/0000-0002-7438-4743 - Javier Del Ángel Caraza

https://orcid.org/0000-0003-4184-2776 - Marco Antonio Barbosa Mireles

https://orcid.org/0000-0001-8139-3114 - José Mauro Victoria Mora

https://orcid.org/0000-0002-9222-7434 - Trinidad Beltrán León 
spp, Ancylostoma spp and Giardia spp were the zoonotic species identified. Conclusions. The results indicate that parks in the conurbed area of Toluca represent a major public health problem as they are a source of zoonotic gastrointestinal parasites of dogs.

Keywords: Parasitosis, Zoonosis, Soil, Green Areas Contamination.

\section{Introducción}

La presencia de parásitos en un área específica está determinada por factores ambientales y por las características propias de estos organismos, lo que permite su sobrevivencia y permanencia en distintas zonas (1). Se ha establecido que además de la transmisión directa, la fuente de infección para hospederos definitivos e intermediarios son los suelos contaminados (2).

La población a estudiar, uso de antiparasitarios, zona geográfica, demografía e implementación de técnicas de laboratorio sensibles, determinan la distribución de parásitos; mientras que la edad, hábitos alimenticios, presencia de animales de compañía en casa y geofagia se asocian a factores de riesgo de los hospederos (2).

La parasitosis se define como una asociación entre dos organismos de distinta especie, en donde la dependencia del parásito respecto al hospedero es metabólica y supone un intercambio mutuo de sustancias (3). Los perros y gatos son hospederos definitivos, intermedios o incidentales de parásitos, lo cual supone un riesgo para la transmisión de enfermedades zoonóticas. Para su estudio los parásitos se han agrupado en tres géneros: nematodos, céstodos y protozoarios; de los cuales se conoce el grado de patogenicidad en el hospedero y cuál es su potencial zoonótico (4).

Existe un riesgo latente de transmisión de parasitosis a humanos, que se deriva del rol que desempeñan los perros y gatos en la dinámica social actual; este riesgo está determinado por la distribución de parásitos en animales de compañía y por factores de riesgo propios de las personas (5).

Un estudio en Italia reporta una contaminación con heces en áreas metropolitanas desde el $41 \%$ hasta el $75 \%$, con prevalencia parasitaria de 35\% (6); en Australia, 38\% de contaminación de áreas y prevalencia de 28.7\% (7); en Colombia, 94.2\% de contaminación y prevalencia de $24.1 \%(8)$; en Chile, contaminación de $37.5 \%$ y prevalencia de $28.4 \%$ (9); lo que indicaría que el suelo es una fuente de transmisión de parásitos tanto a animales de compañía como al humano (1).

El panorama en México es similar. En diversos estudios se ha reportado la contaminación en parque públicos y la prevalencia de parasitosis en las muestras totales como se describe a continuación: en San Cristóbal de las Casas, Chiapas, se reporta una contaminación del 100\% de los parques y 
prevalencia global de 37\% (10); en la Ciudad de México del 75.3\% y 14.6\% (11); Tulyehualco, Estado de México 100\% y 60\% (12); Nezahualcóyotl, Estado de México $100 \%$ y $39 \%$ respectivamente (13). En la zona metropolitana de Toluca los estudios se han enfocado a la población en hospitales veterinarios, centros de control, o muestreos aleatorios en casas habitación; sin que exista una investigación que determine el grado de contaminación de parques públicos y la frecuencia de parásitos en estos espacios.

Es necesario determinar la presencia de parásitos gastrointestinales en parques públicos de la zona conurbada de Toluca y Metepec, para identificar los parásitos frecuentes, conocer el grado de contaminación de parques e identificar las especies parasitarias zoonóticas de la región. Para esto, establecimos los siguientes objetivos: (1) determinar la frecuencia de parásitos gastrointestinales en parques públicos de Metepec y Toluca, Estado de México; (2) determinar la contaminación de parques públicos con parásitos gastrointestinales de perros e edentificar los parásitos con potencial zoonótico.

\section{Materiales y métodos}

\section{Área de estudio y límite de tiempo.}

Las ciudades de Metepec y Toluca se encuentran dentro de la zona metropolitana del Valle de Toluca, con una población estimada de 1.3 millones de habitantes, superficie de $2038 \mathrm{~km} 2$, con una altitud promedio de $2660 \mathrm{msnm}$, con clima predominantemente templado y humedad relativa del 70\% (14). La selección de espacios se realizó de acuerdo al listado oficial de parques y jardines de cada municipio que permiten el acceso a mascotas.

\section{Método de muestreo}

Se muestrearon un total de 27 parques públicos en ambos municipios, recolectando un total de 260 muestras de heces de perro. Las muestras obtenidas se recolectaron en un recorrido equivalente a 1 a 2 horas/ hombre dependiendo del tamańo del parque. El muestreo se realizó en al menos 5 puntos diferentes de cada parque, las heces se mantuvieron en bolsas de polietileno que fueron identificadas con números progresivos, de acuerdo al parque seleccionado y se mantuvieron en refrigeración hasta su procesamiento.

\section{Análisis de las muestras}

La técnica coproparasitoscópica que se seleccionó para procesar las muestras fue la de concentración por flotación fecal (15). Las soluciones utilizadas fueron solución Sheather y sulfato de zinc. Posteriormente se evaluaron microscópicamente con objetivo $10 \mathrm{x}$ y $40 \mathrm{x}$ con un microscopio óptico de campo claro (Primostar, Zeiss, México). Tomándose como positivo las muestras donde se observaron ooquistes, quistes y huevos o larvas de parásitos. Los resultados se registraron en una tabla indicando 
el origen de las muestras, si fueron positivas o negativas, los parásitos identificados, género y potencial zoonótico (de acuerdo a la clasificación de Robertson y Thompson) (4).

\section{Resultados y discusión}

Todos los parques visitados estuvieron contaminados con heces de perro, de los cuales 22 (es decir $81.4 \%$ ) resultaron positivos a estadios parasitarios, lo que indica una alta contaminación de suelos (Figura 1) que corresponde con los datos reportados en Colombia, San Cristóbal de las Casas y la zona conurbada de la Ciudad de México y el Estado de México (8, 10-12). Este dato representa una oportunidad de creación de políticas públicas hacia el control de los perros callejeros con y sin dueño, debido a la alta identificación de especies que son zoonóticas y con las que se encuentra en contacto la población al visitar estos sitios.

La identificación global de parásitos gastrointestinales en las heces fue de 16.5\% (43 muestras), la cual es menor a la reportada en otros municipios del Estado de México como Tulyehualco y Nezahualcóyotl, dónde se observó una prevalencia parasitaria de $60 \%$ y $39 \%$ respectivamente $(12,13)$.

Figura 1. Frecuencias de identificación por especie parasitaria: Ancylostoma spp 18.6\%, Toxocara spp 13.9\%, Cystoisospora spp 13.9\%, Trichomona spp $2.3 \%$. Giardia spp 48.8\%, Sarcocystis spp $2.3 \%$.

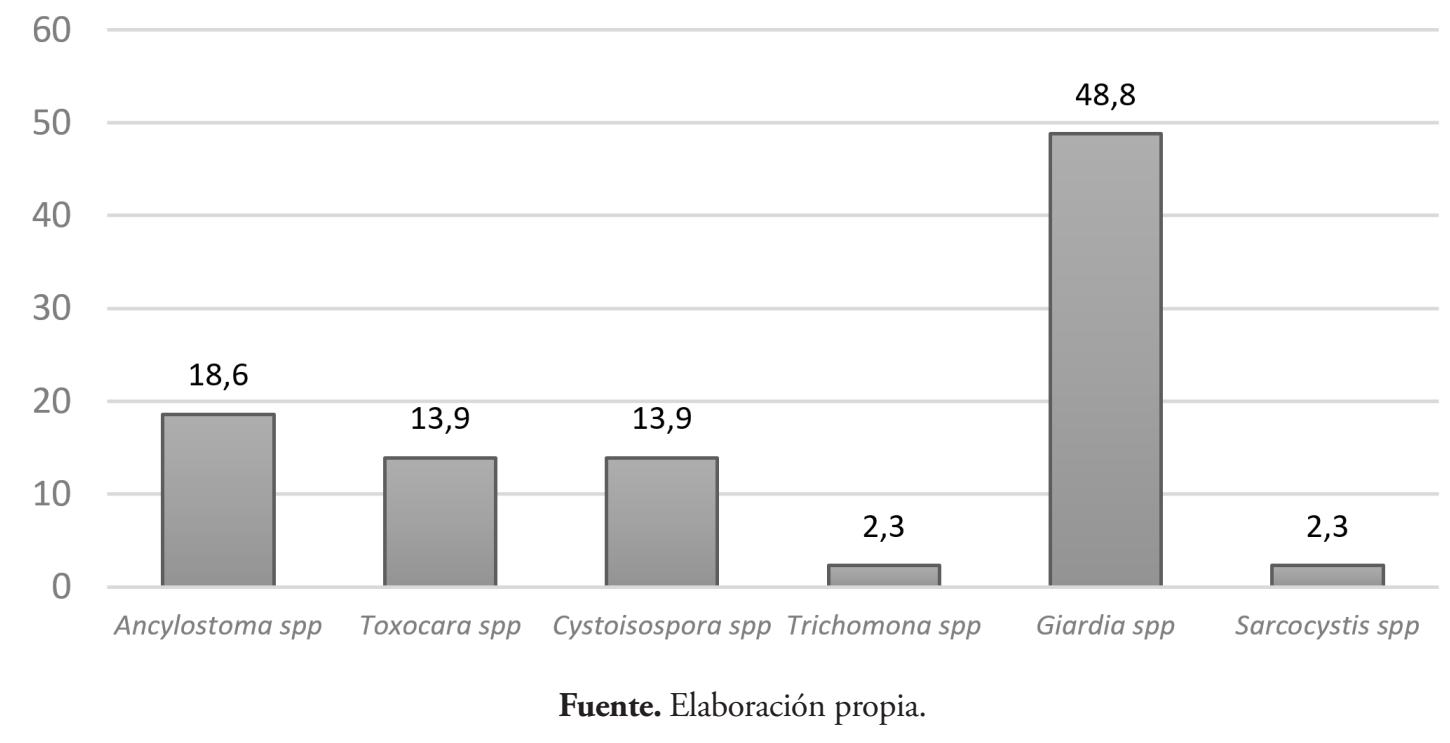

Giardia spp (Figura 2) fue el parásito más frecuentemente observado con $48.8 \%$ de los casos positivos lo que es mayor a lo observado en Australia (22.1\%) o Italia (25.5\%)
$(6,7)$. En México, esta parasitosis no se ha reportado en estudios de contaminación de parques. 
Figura 2. Al centro, 3 quistes de Giardia spp identificadas en las heces de perro provenientes de un parque. Campo claro, 40x

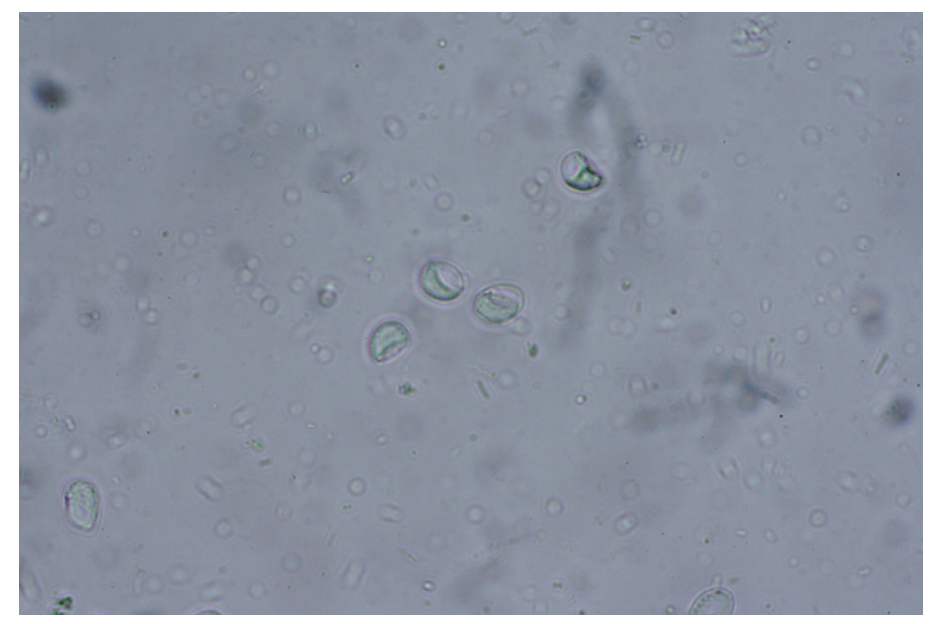

Fuente. Elaboración propia.

$81.4 \%$ de las muestras positivas, resultaron positivas para parásitos con potencial zoonótico; particularmente, Toxocara spp (Figura 3) y Ancylostoma spp (Figura 4), siendo ambas especies reportadas como causantes de larva migratoria subcutánea u ocular; y finalmente, Giardia spp entre las especies encontradas en la zona. Toxocara spp ha sido el parásito zoonótico más investigado en la zona del Estado de México y Distrito Federal por lo que los estudios realizados enfocan sus resultados al diagnóstico de este parásito (11-13). Sin embargo, es importante ampliar las posibilidades diagnósticas para poder identificar otros parásitos zoonóticos como es el caso de nuestro estudio.

Figura 3. Huevos de Toxocara canis identificadas en las heces de perro provenientes de un parque. Campo claro, 40x.

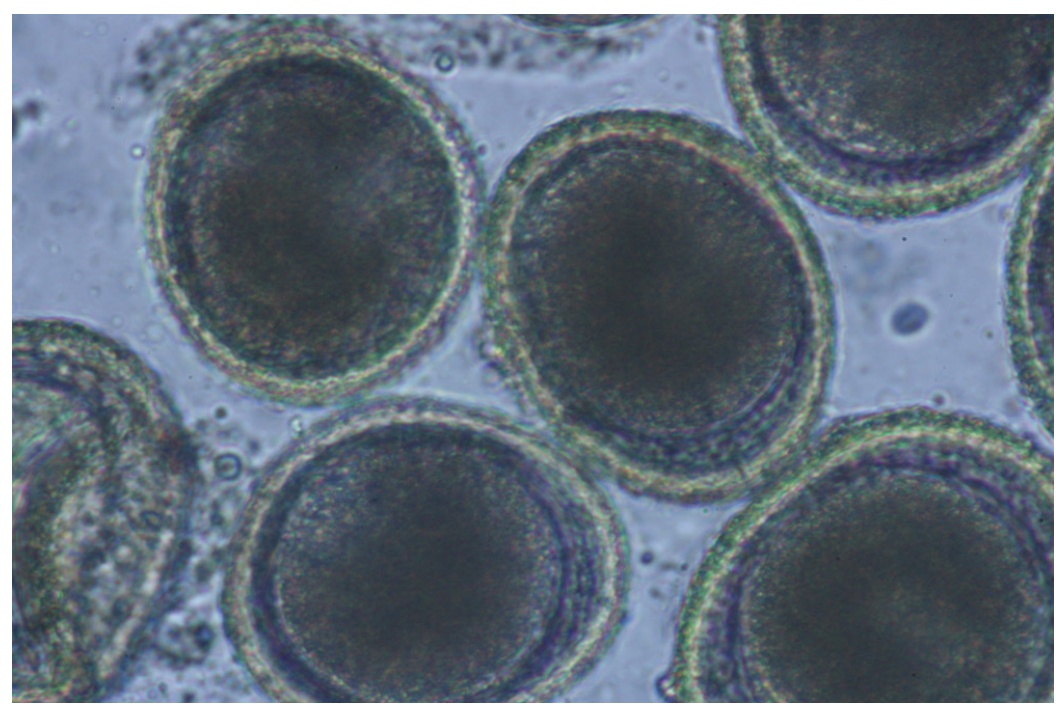

Fuente. Elaboración propia. 
Figura 4. Huevo de Ancylostoma canis identificadas en las heces de perro provenientes de un parque. Campo claro, 40x.

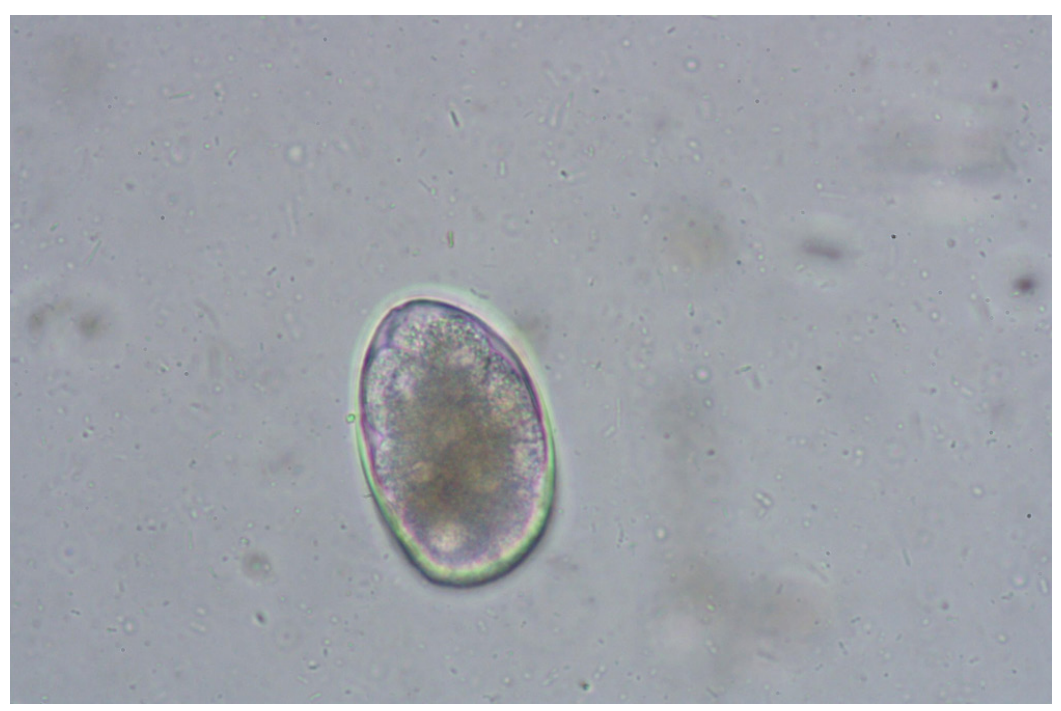

Fuente. Elaboración propia.

\section{Conclusiones}

Este es el primer estudio que se realiza para determinar la contaminación de espacios públicos en la zona conurbada de Toluca y Metepec. Los resultados indican que todos los parques se encuentran contaminados con heces, y en poco más de cuatro de cada cinco parques se identificaron parásitos gastrointestinales.

Las especies parasitarias que se encontraron son: Toxocara spp, Ancylostoma spp y Cystoisospora spp, Sarcocystis spp y Giardia spp Trichomona spp.

Giardia spp fue el parásito más frecuente en la zona. La identificación de esta especie se asocia al uso de técnicas de flotación adicionales como sulfato de zinc y solución Sheather, que permiten su diagnóstico. Concluimos que el uso de más de una técnica coproparasitoscópica, permite ampliar las posibilidades diagnósticas.

$81.3 \%$ de las muestras positivas representaban especies parasitarias zoonóticas: Toxocara spp, Ancylostoma spp y Giardia spp; lo cual asociado a la alta contaminación de los espacios públicos supone un riesgo zoonótico.

\section{Referencias}

1. Overgaauw P, Van Zutphen L, Hoek D, et al. Zoonotic Parasites in fecal samples and fur from dogs and cats in Netherlands. Vet Parasitol 2009;163:115122

2. Robertson ID, Irwin PJ, Lymbery AJ, et al. The role of companion animals in the emergence of parasitic zoonoses. International Journal for Parasitology 2000; 30:1369-1377

3. Quiroz H Parasitología y enfermedades parasitarias de animales domésticos, Edit. Limusa, México, 2008. 
4. Robertson ID, Thompson RC. Enteric parasitic zoonoses of domesticated dogs, Microbes and Infection 2002;4:867-873

5. Machperson CM. The epidemiology and public health importance of toxocariasis: A zoonosis of global importance. International Journal for Parasitology 2013;43:999-1008

6. Zanzani S, Libera G, Scarpa P, et al. Intestinal parasites of owned dogs and cats from Metropolitan and Micropolitan areas; Prevalence, zoonotic risks, and pet owners awarenes in Nothern Italy. BioMed Reserch International 2014;2014; 1-10

7. Bugg R, Robertson I, Elliot D, Thompson C. Gastrointestinal parasites of urban dogs in Perth, Western Australia. The Veterinary Journal 1999;157:295-301

8. Polo T L, Cortés V J, Villamil J C, et al. Contaminación de los Parques Públicos de la localidad de Suba, Bogotá con nemátodos zoonóticos, Rev. Salud Publica 2007;9:550-557

9. Castillo D, Paredes C, Zañartu C, et al. Contaminación ambiental por huevos de Toxocara spp. en algunas plazas y parques públicos de Santiago de chile, 1999; Bol. Chil. Parasitol 2000;55:86-91

10. Martínez BI, Gutierrez CEM, Alpízar SEA, et al. Contaminación parasitaria de heces de perros, recolectadas en la Ciudad de San Cristobal de las Casas, Chiapas, México. Vet. Mex 2008;39:173-180

11. Martínez BI, Fernández PA, Vázquez TO, et al. Frecuencia de Toxocara canis en perros y áreas verdes del sur de la ciudad de México, Distrito Federal, Vet Méx 1998;29:239-244

12. Romero NC, García CA, Mendoza MG, et al. Contaminación por Toxocara spp. En parques de Tulyehualco, México, Revista científica, FCV-LUZ 2009;XIX:253-256

13. Romero NC, Mendoza MD, Bustamante LP y col. Presencia y viabilidad de Toxocara spp en suelos de parques públicos, jardines de casas y heces de pe- rros en Nezahualcóyotl, México. Revista Científica, FCV-Luz 2011;XXI:195-201

14. INEGI, Censo de Población y vivienda, Instituto Nacional de Geografía y Estadística, http://www. censo2010.org.mx. Ultimo acceso 13 Septiembre de 2014.

15. Duorado C, Ferreira J, Talamini A, et al. A new laboratorial method for the diagnosis of gastrointestinal parasites in dogs. Rev. Bras. Parasitol. Vet. Jaboticabal 2013;22:1-5 